\title{
Evolução biológica versus filo cnidaria: panorama dos últimos 10 anos (2010 - 2019) no nordeste do Brasil
}

\section{Biological evolution versus phylum cnidaria: overview of the last 10 years (2010 - 2019) in northeast Brazil}

\author{
Bruno Michael da Silva Pereira ${ }^{1}$, Mayara de Farias ${ }^{2}$ \\ 1 Universidade Federal de Alagoas (UFAL), Maceió, Alagoas, Brasil. ORCID: https://orcid.org/0000-0001-7426-7574 \\ ${ }^{2}$ Secretaria de Educação de Craíbas, Craíbas, Alagoas, Brasil. ORCID: https://orcid.org/0000-0002-1016-3853.
}

Autor para correspondência/Mail to: Bruno Michel da Silva Pereira, brnmichael1@gmail.com

Recebido/Submitted: 01 de agosto de 2020; Aceito/Approved: 15 de setembro de 2020

Copyright (c) 2020 Pereira \& Farias. Todo o conteúdo da Revista (incluindo-se instruções, política editorial e modelos) está sob uma licença Creative Commons Atribuição-NãoComercial-Compartilhalgual 3.0 Não Adaptada. Ao serem publicados por esta Revista, os artigos são de livre uso em ambientes educacionais, de pesquisa e não comerciais, com atribuição de autoria obrigatória. Mais informações em http://revistas.ufpr.br/atoz/about/submissions\#copyrightNotice.

\begin{abstract}
Resumo
Introdução: a temática Evolução Biológica permeia transversalmente todas as áreas do conhecimento das Ciências Biológicas. Ainda assim, é carente de respostas que conectem o nosso presente ao passado, principalmente quando os objetos de estudo são invertebrados, pelo fato do difícil registro das estruturas morfofisiológicas desses organismos. Metodologia: o presente estudo buscou realizar um levantamento na literatura científica brasileira na Google Acadêmico dos últimos 10 anos (2010 - 2019) a respeito de estudos em Evolução relacionados ao filo Cnidaria, no Nordeste do Brasil, utilizando cinco search strings previamente pensadas para condução da busca sobre o tema sugerido. Resultados: foram encontrados 839 documentos e apesar da variação de palavras, depois da triagem, apenas 16 trabalhos foram computados. Trabalhos que tinham como áreas de conhecimento: Paleobiologia, Taxonomia integrativa, Biologia Molecular e Filogeografia. Nos trabalhos encontrados, Anthozoa foi a Classe de Cnidaria mais representativa. O estado de Pernambuco teve a maior contribuição de estudos para a região. Conclusão: ainda que a busca tenha sido de trabalhos dos últimos 10 anos (2010 - 2019), os resultados dão um panorama parcial do real problema que é a falta de estudos que abranjam o tema Evolução, quando se trata de cnidários na região Nordeste do Brasil. Não se exclui a possibilidade da existência de outros trabalhos nesse período, mas com os instrumentos de busca utilizados os resultados demonstraram, mesmo que superficialmente, uma problemática geral, carente de estudos e novos pesquisadores com interesse pela temática, para que as demandas dos conhecimentos da área sejam supridas.
\end{abstract}

Palavras-chave: Evolução Biológica; Filo Cnidaria; Ciências Biológicas.

\begin{abstract}
Introduction: biological Evolution permeates across all areas of knowledge in Biological Sciences. Nevertheless, is in need of answers that connect our present to the past, especially when the objects of study are invertebrates, due to the difficult record of the morphophysiological structures of these organisms. Method: the present study sought to conduct a survey of the Brazilian scientific literature on Google Scholar for the last 10 years (2010 - 2019) regarding studies in Evolution related to the phylum Cnidaria in Northeast Brazil, using five search strings previously thought for conducting the search on the suggested topic. Results: a total of 839 files were found and despite the variation of words, after the screening, only 16 papers were computed. Studies that had as areas of knowledge: Paleobiology, Integrative Taxonomy, Molecular Biology and Phylogeography. In the studies found, Anthozoa was the most representative class of Cnidaria. Pernambuco state had the largest contribution of studies to the region. Conclusions: even though the search was for studies from the last 10 years (2010 - 2019), the results give a partial overview of the real problem, which is the lack of studies covering the theme Evolution, when it comes to cnidarians in the Northeast region of Brazil. The possibility of the existence of other studies in this period is not excluded, but with the search instruments used, the results demonstrated, even if superficially, a general problem, lacking studies and new researchers interested in the theme, so that the demands of knowledge are met.
\end{abstract}

Keywords: Biological evolution; Phylum Cnidaria; Biological Science.

\section{INTRODUÇÃO}

Os cientistas costumam dizer que a Biologia Evolutiva é o eixo transversal que percorre todas as áreas das Ciências Biológicas, atingindo alguns segmentos das ciências exatas e humanidades (Futuyma, 2009; Pigliucci, 2009; Tidon \& Vieira, 2009). Em outras palavras, a Evolução Biológica trata de como teriam ocorrido às transformações gradativas na matéria viva, de modo a possibilitar a vida, bem como dos caminhos percorridos pelos seres vivos ate chegar à diversidade atual (Silva, Santos, \& Dal Pian, 1997).

Esta teoria possui um papel fundamental para a compreensão das demais áreas de conhecimento da Biologia. A teoria da evolução é tida como a mais importante nas Ciências Biológicas, por sua abrangência e seu alcance intelectual e por poder acrescentar uma dimensão extra a história natural do planeta Terra (Ridley, 2006).

Silva, Lavaginini, e Oliveira (2009) abordam que a Evolução Biológica deva ser um eixo integrador entre as diferentes áreas de ensino da Biologia, mas o que se percebe é que, além dessa integração não estar acontecendo, ainda encontramos entraves no ensino de Evolução que esta intrinsecamente relacionada com a formação do professor de Biologia. Autores como Rosa, Muniz, Carneiro, e Goedert (2002), Oleques, Bartholomei-Santos, e Boer (2011) e Villa-Branco Júnior (2000) relatam que a evolução na área da educação tem mostrado dificuldades 
tanto no processo de ensino-aprendizagem como a necessidade de aprimorar e ter formação inicial e continuada para professores da área.

No entanto, o desenvolvimento de trabalhos que abordem a compreensão da Teoria Evolucionista e associações com os diversos grupos biológicos ainda apresentam inúmeras falhas e dificuldades, sendo eminente a necessidade de um maior contato com o tema. E quando direcionado a alguns grupos específicos os resultados são ainda mais desanimadores, conhecimentos estes extremamente necessários para integralizar os dados dos organismos com os das demais áreas dos estudos em Evolução e as Ciências Biológicas em geral (Pigliucci, 2009).

É o que acontece com o filo Cnidaria que é um grupo diverso com aproximadamente 9000 espécies, estruturalmente simples unidos pela habilidade de sintetização de células altamente complexas, as cnidas. Seus membros que incluem corais, hidroides, águas-vivas e anêmonas, são abundantes e comuns no ambiente marinho (Appeltans et al., 2012; Daly et al., 2007). Estes organismos, apesar de primitivos, apresentam inúmeras características evolutivas, sendo os primeiros organismos a desenvolverem uma cavidade digestiva no interior do corpo, um rudimentar sistema nervoso além de algumas características morfológicas evolutivas (Brusca \& Brusca, 2007).

Contudo, muito além do conhecimento geral das características evolutivas e o posicionamento filogenético dos organismos, os estudos em Evolução Biológica representam um papel fundamental para a consolidação de todas as áreas de conhecimento da Biologia (Ridley, 2006). Ainda assim, é carente de respostas que conectem o nosso presente ao passado, principalmente quando os objetos de estudo são invertebrados, pelo fato do difícil registro das estruturas morfofisiológicas desses organismos. Em contra partida, no Brasil, e mais notadamente no Nordeste, existem sítios arqueológicos com registros fósseis de invertebrados primitivos ao decorrer das eras geológicas, além de abrigar a maior área recifal do país e uma das maiores do mundo. Sendo assim, o presente estudo buscou realizar um levantamento na literatura científica brasileira dos últimos 10 anos (2010 - 2019) a respeito de estudos em Evolução relacionados ao filo Cnidaria, no Nordeste do Brasil. A fim de construir um panorama de como este grupo de organismos está sendo estudado e quantificar a produção científica da área em questão.

\section{METODOLOGIA E RESULTADOS}

As buscas dos trabalhos publicados foram realizadas na base de dados da Google Acadêmico, em Julho de 2020, dos últimos 10 anos $(2010$ - 2019) de publicações em português sobre o tema sugerido. Partindo do pressuposto de que as publicações em português facilitariam o acesso aos pesquisadores brasileiros, por conveniência da língua materna. A Google Acadêmico (bases de dados e agregadores) possui um grande acervo de trabalhos nacionais e da América Latina o que atendem as necessidades desta pesquisa.

Foram escolhidos cinco "search strings" previamente pensadas para conduzir melhor a busca de publicações que englobaram o tema escolhido. Os resultados da busca estão representados na Tabela 1 com o número de artigos encontrados para cada combinação de palavras utilizadas. Para refinar a busca dos trabalhos publicados, foi utilizado o operador lógico booleano "E", na Google Acadêmico para acessar em todas as plataformas associadas a esta base documentos com a temática proposta, para que em qualquer hipótese, dependendo do pressuposto de pesquisa da base, fossem encontrados documentos que contivessem o tema da pesquisa. Esses operadores booleanos têm a função de criar uma interseção entre as palavras para mostrar todos os trabalhos que contenham todas as palavras inseridas, a fim de se ter uma amplitude maior nas buscas, quando relacionadas às palavras utilizadas. Também foi utilizado o Caractere asterisco $\left(^{*}\right)$, utilizado no final dos termos pretendidos para aumentar o alcance da busca, para em vez de pesquisar apenas "Característica Evolutiva", por exemplo, com o * (Característica* Evolutiva*) a busca se ampliou também para "CaracterísticaS EvolutivaS", da mesma forma com o termo "caracter*" com a variação do plural. As aspas ("'") foram utilizadas para indicar a frase exata que se pretendia buscar na combinação de palavras utilizadas, para que estas venham sempre juntas e exatamente na forma que foi inserida.

As informações dos trabalhos encontrados foram obtidas através de uma leitura sistemática do título, resumo, palavras chave e resultados de todos os arquivos utilizados, depois de uma prévia triagem das publicações para excluir os artigos que em seu conteúdo não estavam relacionados a estudos que englobem a temática evolução envolvendo cnidários. 


\begin{tabular}{lc}
\hline Palavras utilizadas para a busca & No de trabalhos $^{\circ}$ \\
\hline Teoria* evolução* E Cnidaria E "Nordeste do Brasil" & 222 \\
Evolução E Cnidaria E "Nordeste do Brasil" & 142 \\
Característica* evolutiva* E Cnidaria E "Nordeste do Brasil" & 157 \\
Seleção natural E Cnidaria E "Nordeste do Brasil" & 219 \\
Variação de caracter* E Cnidaria E "Nordeste do Brasil" & \\
\hline
\end{tabular}

Tabela 1. Número de trabalhos encontrados em 10 anos com a utilização das cinco searchs strings na base de dados da Google Acadêmico 2010 - 2019.)

Fonte: Dados da pesquisa (2020).

Foram encontrados 839 documentos. Apesar da variação de palavras, depois da triagem, nos últimos 10 anos, apenas 16 trabalhos foram computados, pois, apenas estes obtinham as informações que puderam ser utilizadas no estudo (Tabela 2). Apesar de esse baixíssimo número de registros, foi possível observar uma tendência maior de interesse com essa temática, nos anos de 2012, 2014, 2015 e 2017, com inexistência de trabalhos nos anos 2010, 2016 e 2018. Ainda assim, a maioria dos trabalhos realizados nesse período é considerada "literatura cinzenta", sendo eles: oito Dissertações, duas Teses e um resumo em congresso. Trabalhos que apesar de grande relevância, sua maior fonte de divulgação e interna, em Instituições de Nível Superior. Sendo apenas cinco deles artigos científicos, trabalhos que pela via de divulgação têm um alcance maior.

Com relação aos locais de coleta de dados, o local em que houve o maior número de estudos realizados foi o estado de Pernambuco, com sete no total. Bahia e Ceará tiveram quatro estudos cada. Alagoas e Rio Grande do Norte com três trabalhos em cada estado. Seguidos de Maranhão e Paraíba, com um estudo. Em alguns dos estudos foram realizadas coletas em mais de um estado, o que aumentou a abrangência de pesquisas na região. A distribuição desses trabalhos realizados reflete que dentre as Universidades as quais os pesquisadores que desenvolveram esses estudos estão vinculados a Universidade Federal de Pernambuco desenvolveu $59 \%$ do total de trabalhos com a temática, seguidamente a Universidade Federal da Bahia com 17\% e Universidade Federal de Alagoas, Universidade Federal Rural de Pernambuco, Universidade Federal do Rio Grande do Norte e Universidade de São Paulo contribuíram com 6\% cada. Esta última, a única Universidade que esta fora do Nordeste realizando um trabalho de caráter filogeográfico. Todos os resultados foram sumarizados, podendo ser observados na Tabela 2. Aditivamente, traçando o perfil dos pesquisadores destas Instituições, com relação à produção do conhecimento da temática foi possível observar que $62,5 \%$ eram do sexo feminino.

\begin{tabular}{cclllll}
\hline Artigo & \multirow{2}{*}{ Ano } & Universidade & Local de coleta & $\begin{array}{l}\text { Tipo de } \\
\text { trabalho }\end{array}$ & Classe & Área \\
\hline 1 & 2011 & USP & CE & Artigo & Cubozoa & Filogeografia \\
2 & 2012 & UFPE & CE & Dissertação & Cnidaria* & Paleobiologia \\
3 & 2012 & UFPE & BA, CE, RN, MA, AL, PB & Dissertação & Anthozoa & Tax. integrativa \\
4 & 2012 & UFPE & PE, AL, BA & Dissertação & Anthozoa & Tax. integrativa \\
5 & 2013 & UFRPE & RN, PE & Resumo & Anthozoa & Evolução \\
6 & 2014 & UFPR & PE & Tese & Anthozoa & Evolução \\
7 & 2014 & UFRN & RN & Dissertação & Cnidaria* & Paleobiologia \\
8 & 2014 & UFPE & PE & Dissertação & Anthozoa & Tax. integrativa \\
9 & 2015 & UFBA & BA & Dissertação & Anthozoa & Evolução \\
10 & 2015 & UFPE, UFAL & Al & Artigo & Anthozoa, Hydrozoa & Paleobiologia \\
11 & 2015 & UFPE & PE & Tese & Anthozoa & Bio. Molecular \\
12 & 2015 & UFPE & PE & Dissertação & Anthozoa & Evolução \\
13 & 2017 & UFBA & BA & Dissertação & Anthozoa, Hydrozoa & Paleobiologia \\
14 & 2017 & UFPE & CE & Artigo & Anthozoa & Paleobiologia \\
15 & 2017 & UFPE & PE & Artigo & Cnidaria* & Paleobiologia \\
16 & 2019 & UFBA & BA & Artigo & Anthozoa, Hydrozoa & Paleobiologia \\
\hline
\end{tabular}

Tabela 2. NIndicadores de produção científica na temática evolução biológica com relação ao filo Cnidaria na Google Acadêmico (2010 - 2019$).$ Fonte: Dados da pesquisa (2020).

Ainda com relação aos pesquisadores em se tratando de grupos de pesquisa, quando pesquisados os temas de interesse "invertebrados marinhos", "Cnidaria" ou "bentos" no Diretório dos Grupos de Pesquisa do CNPq para a região Nordeste, foram encontrados 21 grupos de pesquisa que desenvolvem trabalhos com a temática proposta por este estudo de forma geral.

As áreas de conhecimento que foram enfoque das pesquisas desenvolvidas, majoritariamente foram estudos em Paleobiologia, sete trabalhos. Evolução, como um tema geral, foram encontrados quatro trabalhos; Taxonomia Integrativa, três estudos; e Biologia Molecular e Filogeografia, com um trabalho cada. Dentre as quatro classes 
do filo Cnidaria, apenas três foram citadas como objeto de estudo nos trabalhos encontrados, Anthozoa foi a mais representativa, com doze estudos; Hydrozoa, com três e Cubozoa com um estudo. Ficando a classe Scyphozoa sem nenhuma representatividade. Apesar de a maioria dos estudos terem sido realizados com a classe Anthozoa, o gênero Millepora sp. foi o mais estudado, representante da classe Hydrozoa.

\section{CONCLUSÕES}

Ainda que a busca tenha sido de trabalhos dos últimos 10 anos (2010 - 2019), os resultados dão um panorama parcial do real problema que é a falta de estudos que abranjam o tema Evolução e suas áreas correlatas, quando se trata de cnidários na região Nordeste do Brasil. Não se exclui a possibilidade da existência de outros trabalhos nesse período, mas com as limitações da pesquisa e os instrumentos de busca utilizados os resultados demonstraram, mesmo que superficialmente, uma problemática geral, carente de estudos e interesse pela temática por parte dos pesquisadores, mostrando que dos nove estados do Nordeste em apenas sete desenvolveram estudos com o tema proposto, o que reflete a problemática que a literatura descreveu, criando as falhas e dificuldades no entendimento da Evolução e mais especificamente quando relacionada ao filo Cnidaria, que foi o enfoque desta pesquisa.

Ainda assim, existem grupos de pesquisa cadastrados no CNPq no Nordeste do Brasil reconhecidos, mundialmente, por suas contribuições para a produção do conhecimento acerca dos invertebrados marinhos na costa brasileira, mas, apesar de essa grande contribuição, devido à abrangência dos grupos de invertebrados, torna-se uma tarefa carente de novos pesquisadores para que as demandas dos conhecimentos da área sejam supridas. Resultados como os obtidos neste estudo ajudam a nortear jovens pesquisadores para a elucidação dessas lacunas, como fonte de inspiração e até mesmo para a descoberta e soluções de novos problemas.

A análise e a sumarização de dados têm esse papel fundamental na exploração e na descoberta de novos rumos. Transformar os dados sejam eles locais ou partindo de metadados, em conhecimento é uma ferramenta que possibilita uma fácil e rápida comunicação na comunidade científica. Essa integração entre os conhecimentos adquiridos pode, ainda, de maneira mais abrangente orientar e dar suporte para uma abordagem mais global e assertiva na busca de respostas. Foi possível observar que apesar do enfoque direcionado deste trabalho, por tratar de um único filo, abre-se uma janela com um leque de inúmeras possibilidades, para que outros grupos sejam analisados, com as mais diversas abordagens ou áreas do conhecimento. 


\section{REFERÊNCIAS}

Appeltans, W., Ahyong, S. T., Anderson, G., Angel, M. V., Artois, T., Bailly, N., ... Berta, A. (2012). The magnitude of global marine species diversity. Current Biology, 22(23), 2189-2202. doi: 10.1016/j.cub.2012.09.036.

Brusca, G. J., \& Brusca, R. C. (2007). Invertebrados. (2a. ed.). Rio de Janeiro: Guanabara-Koogan.

Daly, M., Brugler, M. R., Cartwright, P., Collins, A. G., Dawson, M. N., Fautin, D. G., ... Stake, J. L. (2007). The phylum cnidaria: A review of phylogenetic patterns and diversity 300 years after linnaeus. Zootaxa, 1668(1), 243-263. Recuperado de https://www.biotaxa.org/Zootaxa/ article/view/zootaxa.1668.1.11/0.

Futuyma, D. J. (2009). Biologia evolutiva. (3a. ed.). Ribeirão Preto: Funpec.

Oleques, L. C., Bartholomei-Santos, M. L., \& Boer, N. (2011). Evolução biológica: percepções de professores de biologia. Revista Electrónica de Ensenanza de Las Ciencias, 10(2), 243-263.

Pigliucci, M. (2009). An extended synthesis for evolutionary biology. the year in evolutionary biology. Annals of the New York Academy of Sciences, 1168, 218-228.

Ridley, M. (2006). Evolução. (3a. ed.). Porto Alegre: Artmed.

Rosa, V., Muniz, E. C. N., Carneiro, A. P. C., \& Goedert, L. (2002). O tema evolução entre professores de biologia não licenciados - dificuldades. In Anais do viii encontro perspectivas do ensino de biologia. São Paulo, Brasil.

Silva, Lavaginini, T. C., \& Oliveira, R. R. d. (2009). Propostas de uma estratégia didática para o ensino de evolução biológica no ensino médio. In Anais do xi encontro nacional de pesquisa em educação em ciências. Florianópolis, Brasil. Recuperado de http://gpeqsc.com.br/guaipira/artigos/ 2009 _ _ENPEC.18.pdf.

Silva, Santos, A. K. P., \& Dal Pian, M. C. (1997). Para pensar e ensinar a teoria da evolução. In Anais do i encontro nacional de pesquisa em educação em ciências. Águas de Lindóia, Brasil.

Tidon, R., \& Vieira, E. (2009). O ensino da evolução biológica: um desafio para o século xxi. Com Ciência(107).

Villa-Branco Júnior, V. B. E. (2000). Prática e teoria de evolução para professores do ensino médio. In Anais do vii encontro perspectivas do ensino de biologia. São Paulo, Brasil.

Como citar este shortpaper (APA):

Pereira, B. M. da S. \& Farias, M. de (2020). Evolução biológica versus filo cnidaria: panorama dos últimos $10 \operatorname{anos}(2010$ - 2019) no nordeste do Brasil. AtoZ: novas práticas em informação e conhecimento, 9(2), 108 - 112. Recuperado de: http://dx.doi.org/10.5380/ atoz.v9i2.75584 